Electronic version of an article published as [Annals of Operations Research, 2007. Vol. 155, No. 1, p. 5-23] [DOI: http://dx.doi.org/ 10.1007/s10479-007-0211-3]

(C) [copyright Springer] 


\title{
Planning production and working time within an annualised hours scheme framework
}

\author{
Albert Corominas; Amaia Lusa; Rafael Pastor* \\ Research Institute (IOC) / Engineering school (ETSEIB) \\ Universitat Politècnica de Catalunya (UPC); Barcelona; Spain
}

Corresponding author: Amaia Lusa, Research Institute IOC, Av. Diagonal 647 (edif. ETSEIB), p.11, 08028 Barcelona, Spain; Tlf. + 349340117 05; Fax. + 34934016605 ; email: amaia.lusa@upc.edu 


\begin{abstract}
Production flexibility is essential for industrial companies that have to deal with seasonal demand. Human resources are one of the main sources of flexibility. Annualising working hours (i.e., the possibility of irregularly distributing the total number of working hours over the course of a year) is a tool that provides organisations with flexibility; it enables a firm to adapt production capacity to fluctuations in demand. However, it can involve a worsening of the staff's working conditions. To take this into account, the planning and scheduling of working time should comply with constraints derived from the law or from a collective bargaining agreement. Thus, new and more difficult working-time and production planning and scheduling problems are arising. This paper proposes two mixed-integer linear program models for solving the problem of planning the production, the working hours and the holiday weeks of the members of a human team operating in a multi-product process in which products are perishable, demand can be deferred and temporary workers are hired to stand in for employees. The results of a computational experiment are presented.
\end{abstract}

Keywords: human resources, manpower planning, annualised hours, integer programming. 


\section{Introduction}

At the present time, production flexibility is essential for industries that have to deal with seasonal demand. Human resources are one of the main sources of flexibility. Annualising working hours (AH) consists in hiring workers for a certain number of hours per year and distributing those hours irregularly over the course of the year to deal with fluctuations in demand. It allows a better use of potential capacity: one can better adapt capacity to demand throughout the year and therefore reduce inventory levels and lost demand (and their associated costs).

However, AH often involves a worsening of the staff's working conditions (an irregular schedule may affect a worker's private life) and the need to solve a complicated problem in planning working time. To minimise the negative consequences for workers, the annualisation of working time must be negotiated and may be accompanied by some sort of compensation or incentive: a reduction in annual working time, additional holidays or financial compensation. At the same time, constraints derived from the law or from a collective bargaining agreement must be respected to avoid excessively overburdening workers during long high-demand periods.

The practice of annualising hours is a source of flexibility of ever greater importance and the number of firms that are signing $\mathrm{AH}$ contracts is rising. However, the manpower planning and scheduling problems raised by AH have not been widely studied up to the present. Some other authors deal with different versions of the problem (see, for example, Hung, 1999a-b; Gonçalves and Marçola, 2001; Chan and Weil, 2002 and Azmat et al., 2004a-b), but most papers (for example, Clutterbuck 1982, Lynch 1985, Curran 1992, MacMeeking 1995 and Mazur 1995) discuss AH only from a qualitative point of view.

Corominas et al. (2004a, 2005a) discuss from a qualitative point of view the characteristics of the planning problem and propose a classification scheme that gives rise to thousands of different cases (the authors have already solved several of the types of problems, the most common ones). Corominas et al. (2002) use MILP to solve a problem of planning staff working hours using an annual horizon and two hierarchical categories of workers, minimising the cost due to overtime and temporary workers, and regularising the distribution of working time for each worker over the course of the year. Corominas et al. (2004b) deal with the problem of planning annual working hours in which the weekly number of working hours for any worker must fall within a previously agreed interval. Both papers plan working hours but not production because a service centre problem is considered. Finally, in 
Corominas et al. (2005b) a MILP model is used to establish a framework for an annualised hours agreement. In all such cases, in spite of the large size of the models, MILP has been shown to be an efficient tool for solving this kind of problem.

By solving working time and production planning in annualised hours scenarios, one can see how capacity is adapted to demand. Also, if an AH system is compared to a traditional system (regular working hours), one can see how the costs due to overtime, temporary workers, subcontracting, lost demand and—in some cases—inventory are reduced.

Corominas et al. (2005b) deals with the problem of planning the production and the working time of the members of a human team involved in a multi-product process under an annualised hours agreement. The main characteristics of the problem are: (i) employees take their holidays simultaneously and these are fixed beforehand; (ii) the process needs the simultaneous presence of all team members, so production stops when workers are on holidays; (iii) demand can be deferred for a finite time; (iv) there exist conditions affecting the distribution of working time over the year.

In this paper more possibilities are considered in order to make it more general and thus appropriate for a great range of companies. The main differences between the problem dealt in this paper and the one dealt in Corominas et al. (2005b) are the following: (i) employees do not take their holidays simultaneously; (ii) employees holidays may be fixed beforehand or may be part of the problem solution (this gives rise to two different models); (iii) production does not stop, so in some weeks temporary workers may stand in for those in holidays; (iv) the products are perishable. These assumptions together make this problem one of the most complicated cases among those classified in Corominas et al. (2004a).

Whilst the above problem is quite common, it is not to be forgotten that in many companies workers take their holidays simultaneously and the production is then stopped (though demand still exists). To include most real world situations, a second version of the problem is solved, whose main differences from the first are: (i) employees take their holidays simultaneously, and (ii) when workers are on holidays, there is no production (no temporary workers are hired) but there is demand.

The rest of the paper is organised as follows: Section 1 introduces the first version of the problem; Section 2 and 3 describe the two models (the one taking holiday weeks as a data and the one including their determination in it) corresponding to the first version; Section 4 introduces the second version of 
the problem and gives the guideline to formulate the models corresponding to the second version; Section 5 describes the computational experiment and shows the main results of a specific case; and Section 6 contains the main conclusions.

\section{Production planning under annualised hours (workers do not take their holidays simultaneously)}

The planning problem consists in determining the weekly production and the weekly number of working hours for the members of a human team involved in an industrial process and for each week of the planning horizon (a year, for instance), with the objective of optimising a utility function (in this case, the profit). It is assumed that there are different products to plan and their forecasted demand is known.

It is assumed that the process requires the simultaneous intervention of all team members, so in every week all workers must do the same working hours. The number of workers in the team is required to be constant. Thus, if the workers do not take their holidays simultaneously, when an employee is on holiday a temporary worker stands in for her or him. It is also considered that productivity may decrease depending on the presence of temporary workers in the team. Furthermore, the number of temporary workers in any week is upper bounded in order to avoid a loss of quality.

When there is insufficient inventory to respond to demand, the company can defer it (within the same year) but only for a finite time, so there may be lost demand. When demand is deferred, its corresponding income may be less than the habitual income, depending on the delay.

The products are assumed to be perishable so they can be stored only for a finite time. The income may depend on whether the product is near to perishing or not. Furthermore, inventory holding costs may depend on the age of the product (for example, because special measures must be taken in order to keep the products in good condition).

The conditions to be fulfilled by the solution may come from a collective bargaining agreement between management and workers or from a legal resolution, such as the French Aubry II Law, which 
permits an irregular distribution of hours throughout the year, according to certain rules (see MES, 2004). Because it is impossible to establish an exhaustive list of conditions a priori, the most common conditions are considered to build a basic model for the problem (some of these were found in collective bargaining agreements and some were determined through real cases). To adapt this model to a specific case, constraints can be added or deleted.

As an example, French law requires the weekly number of working hours to fall within an interval defined by a lower and an upper bound. The annual total of ordinary working hours is fixed and annual overtime and the average weekly working hours for any set of twelve consecutive weeks must both be upper bounded (it is assumed that this condition applies only to sets of twelve consecutive non-holiday weeks).

To consider the workers' working conditions, the collective agreement may also state that a working week can be considered "weak" or "strong", depending on the number of working hours (for example, no more than 30 hours/week and more than 44 hours/week respectively). The annual number of "weak" working weeks is lower bounded (for example, 10 weeks) and the annual number of "strong" working weeks is upper bounded (for example, 15 weeks).

In most situations holiday weeks are determined separately from the working hours, but in some cases determining them could be part of the problem. Obviously, the company can increase the profit by choosing the best holiday weeks for each employee according to some agreed conditions, and may offer some kind of compensation in exchange.

The characteristics of the problem are summarised as follows:

- There are different products and their forecasted demand is known.

- $\quad$ The products may be perishable (can be stored for a finite time).

- At every week, all workers (except of those that are on holidays) must perform the same working hours.

- Workers do not take their holidays simultaneously. Each worker has two holiday times, with a certain number of consecutive holiday weeks in each of them.

- Holiday weeks may be considered previously agreed (model 1) or are to be determined (model 2). 
- When a worker is on holiday a temporary worker stands in for him.

- The presence of temporary workers in the team may cause a reduction in productivity.

- The number of temporary workers in the team in any week is upper bounded.

- The annual number of ordinary working hours is fixed. Additional hours are considered overtime.

- Annual overtime is upper bounded.

- The weekly number of working hours is lower and upper bounded.

- The average working hours for a group of $L$ non-holiday consecutive weeks cannot be greater than $h_{L}$ hours/week.

- The annual number of "weak" working weeks is lower bounded (a week is "weak" if the number of working hours is no greater than $h_{B}$ hours).

- The annual number of "strong" working weeks is upper bounded (a week is "strong" if the number of working hours is greater than $h_{A}$ hours).

- $\quad$ The company can defer the demand (the delay is upper bounded, so there may be lost demand).

- Profit is to be maximised, considering the incomes (that may depend on the age of the product and the delay in delivering), overtime cost, temporary personnel cost, inventory holding cost (which may depend on the age of the product), lost demand costs and production costs.

These conditions can be modelled as linear constraints.

\section{Model AHFH (annualised hours and fixed holidays)}

In a specific week, the team is formed by the workers that are not on holidays and the temporary workers that stand for those on holidays. Since the process needs the simultaneous intervention of all workers, each member of the team has to work the same hours.

We make use of the following notation.

\section{Data:}


$N T_{t}$

Q

$t p_{q}$

$s t_{q j 0}$

$H_{i}$

$M Y_{i}$

$h m_{t}, h M_{t}$

$L, h_{L}$

$A_{i}, h_{A}$

$B_{i}, h_{B}$

$d_{q t}$

td

$T P_{q}$

$\rho_{q t}$

CE

CT

$C P_{q}$

$C S T_{q t}$ set of workers.

number of weeks of the planning horizon (usually 52).

set of available weeks of the worker $i$ (the weeks of the planning horizon excluding holiday weeks), $\forall i \in W$.

number of temporary workers in week $t(\mathrm{t}=1, \ldots, T)$.

number of products.

maximum number of weeks that product $q$ can be stored $q(\mathrm{q}=1, \ldots, Q)$.

inventory of product $q$ at the beginning of the planning horizon with an age of $j$ weeks $\left(q=1, \ldots, Q ; j=1, \ldots, t p_{q}\right)$.

annual number of ordinary working hours of worker $i(\forall i \in W)$.

maximum annual overtime for worker $i(\forall i \in W)$.

lower and upper bound for the weekly number of working hours.

for each worker, the average working hours in a group of $L$ consecutive working weeks cannot be greater than $h_{L}$ (in that case, $L=12$ and $h_{L}=44$ hours).

$A_{i}$ is the maximum number of "strong" weeks (i.e., weeks with a number of working hours greater than $\left.h_{A}, h m_{t} \leq h_{A} \leq h M_{t}\right)$ for worker $i(\forall i \in W)$.

$B_{i}$ is the minimum number of "weak" weeks (i.e., weeks with a number of working hours not greater than $\left.h_{B}, h m_{t} \leq h_{B} \leq h M_{t}\right)$ for worker $i(\forall i \in W)$.

forecasted demand for product $q$ in week $t(\mathrm{q}=1, \ldots Q ; \mathrm{t}=1, \ldots, T)$.

maximum number of weeks that a demand can be deferred.

time (in hours) needed to prepare the process to produce product $q(\mathrm{q}=1, \ldots, Q)$. If this time depends on the production sequence, an average time is to be considered.

productivity (in units/hour) for product $q$ in week $t(\mathrm{q}=1, \ldots, Q ; \mathrm{t}=1, \ldots, T)$. This value may depend on the week (for example, in Christmas the productivity uses to be lower than the usual) and the number of temporary workers.

cost of overtime (it is assumed to be equal for all workers).

cost of an hour of temporary personnel.

unit cost for product $q(\mathrm{q}=1, \ldots, Q)$; personnel costs are not included.

weekly inventory holding cost of one unit of product $q$ with an age of $t$ weeks $\left(\mathrm{q}=1, \ldots, Q ; \mathrm{t}=1, \ldots, t p_{q}\right)$. 


\begin{abstract}
$C R_{q} \quad$ cost of one unit of lost demand of product $q(\mathrm{q}=1, \ldots, Q)$.
$C K_{q} \quad$ unit cost of eliminating product $q(\mathrm{q}=1, \ldots, Q)$. If there were inventory at the beginning of the planning horizon and the demand were small, some products might perish and have to be thrown away. Depending on the kind of product this could involve some cost.

$I_{q j t}$ income of one unit of product $q(\mathrm{q}=1, \ldots, Q)$ with an age of $j$ weeks $\left(\mathrm{j}=0, \ldots, t p_{q}\right)$ served with a delay of $t$ weeks $(\mathrm{t}=0, \ldots, t d)$; the costs of deferring are assumed to be included in this parameter and it is thus assumed that $I_{q, j, t-1}>I_{q, j, t}>I_{q, j, t+1}$.
\end{abstract}

\title{
Variables:
}

$X_{t}$

$y_{i}$

$a_{t} \in\{0,1\}$

$b_{t} \in\{0,1\}$

$p_{q t}$

$e_{q t} \in\{0,1\} \quad$ indicates whether product $q$ is produced in week $t(\mathrm{q}=1, \ldots, Q ; \mathrm{t}=1, \ldots, T)$

non-negative real variable that indicates the number of working hours of each member of the team in week $t(\mathrm{t}=1, \ldots, T)$.

non-negative real variable that indicates the annual overtime for worker $i(\forall i \in W)$.

if the number of working hours in week $t$ is greater than $h_{A}$ (the week is "strong"), then these variables take value $1(\mathrm{t}=1, \ldots, T)$.

if the number of working hours in week $t$ is greater than $h_{B}$ (the week is not "weak"), then these variables take value $0(\mathrm{t}=1, \ldots, T)$.

non-negative real variable that indicates the quantity of product $q$ produced in week $t$ $(\mathrm{q}=1, \ldots, Q ; \mathrm{t}=1, \ldots, T)$.

$s t_{q j t}$

non-negative real variable that indicates the inventory of product $q$, with an age of $j$ weeks, at the end of week $t\left(\mathrm{q}=1, \ldots, Q ; \mathrm{j}=1, \ldots, t p_{q} ; \mathrm{t}=1, \ldots, T-1\right)$. The variable $s t_{q j T}$ is not defined because at the end of the planning horizon the inventory is assumed to be equal to 0 . It is assumed that the age of a unit produced in a week, at the end of the same week, is 1 . To consider initial conditions $s t_{q j t}, \forall j \in\left[t+1, t p_{q}\right]$ and $\forall t \in\left[1, t p_{q}-1\right]$ is defined if and only if $s t_{q, j-t, 0}>0$; for example, the variable $s t_{q, 3,2}$, indicating the inventory of product $q$ with an age of 3 weeks at the end of week 2 cannot exist if at the beginning of the planning horizon there is no inventory with an age of 1 week (i.e., if $\left.s t_{q, 1,0}=0\right)$.

non-negative real variable that indicates the quantity of the demand of product $q$ that should be delivered in week $s$, is served in week $t$ and has been produced in week $j$ 
$\left(\mathrm{q}=1, \ldots, Q ; \mathrm{s}=1, \ldots, T ; \mathrm{j}=\mathrm{s}-t p_{q}, \ldots, \min (\mathrm{s}+t d, T) ; \mathrm{t}=\max (\mathrm{s}, j), \ldots, \min \left(j+t p_{q}, \mathrm{~s}+t d, T\right)\right)$. To consider initial conditions, $v_{q s j t}\left(\mathrm{q}=1, \ldots, \mathrm{Q} ; \mathrm{s}=1, \ldots, T ; \forall \mathrm{j} \leq 0 ; \mathrm{t}=\max (\mathrm{s}, j), \ldots, \mathrm{min}\left(j+t p_{q}\right.\right.$, $s+t d, T)$ ) is defined if and only if $s t_{q, 1-j, 0}>0$; for example, the variable $v_{q, 1,-1,1}$, indicating the quantity of product $q$ that should be delivered in $s=1$ that has been produced in $\mathrm{j}=-$ 1 and that has been served in $t=1$, must be equal to 0 if at the beginning of the planning horizon there is no inventory with an age of 2 weeks (i.e., if $s t_{q, 2,0}=0$ ); thus, $\mathrm{j}=0$ corresponds to week 52 of the previous year, $\mathrm{j}=-1$ to week 51 , and so on.

$r_{q t}$ non-negative real variable that indicates the quantity of demand of product $q$ corresponding to week $t$ that is not served and lost $(\mathrm{q}=1, . ., Q ; \mathrm{t}=1, \ldots, \mathrm{T})$.

$n v_{q s} \quad$ non-negative real variable that indicates the quantity of product $q$ produced in week $s$ (week belonging to the previous year) that must be thrown away because it has perished: $\mathrm{q}=1, \ldots, Q ; \mathrm{s}=1-t p_{q}, \ldots, 0 \mid\left(s t_{q, 1-\mathrm{s}, 0}>0\right) ; \mathrm{s}=0$ corresponds to week 52 of the previous year, $s=-1$ to week 51 , and so. Obviously, it is not necessary to define this variable for weeks belonging to the planning horizon since in any optimal solution production that would be eliminated is not produced.

\section{Model:}

(1)

$$
\begin{aligned}
{[M A X] Z=} & \left(\sum_{q=1}^{Q} \sum_{s=1}^{T} \sum_{\forall j \in\left[s-t p_{q}, \min (s+t d, T)\right] ı\left[s t_{q, 1-j, 0}>0, \forall j \leq 0\right]} \sum_{t=\max (s, j)}^{\min \left(j+t p_{q}^{, s+t d, T)}\right.} v_{q s j t} \cdot I_{q, t-j, t-s}\right)- \\
& C E \cdot \sum_{\forall i \in W} y_{i}-C T \cdot \sum_{t=1}^{T} N T_{t} \cdot x_{t}-\left(\sum_{q=1}^{Q} \sum_{\forall s \in\left[1-t p_{q}, 0\right] \backslash\left[s t_{q, 1-s, 0}>0\right]} C K_{q} \cdot n v_{q s}\right)- \\
& \sum_{q=1}^{Q}\left(\sum_{t=1}^{T}\left(C P_{q} \cdot p_{q t}+C R_{q} \cdot r_{q t}\right)+\sum_{t=1}^{T-1}\left(\sum_{\forall j \in\left[1, t p_{q}\right] \backslash\left[j \leq t \vee\left(s t_{q, j-t, 0}>0 \wedge j \geq t+1\right)\right]} C S T_{q j} \cdot s t_{q j t}\right)\right)
\end{aligned}
$$

(2) $\sum_{\forall t \in S_{i}} x_{t}=H_{i}+y_{i}$

(3)

$$
\sum_{\forall j \in\left[s-t p_{q}, \min (s+t d, T)\right] \mid\left[s t_{q, 1-j, 0}>0, \forall j \leq 0\right]} \sum_{t=\max (s, j)}^{\min \left(j+t p_{q}, s+t d, T\right)} v_{q s j t}+r_{q s}=d_{q s} \quad(q=1, \ldots, Q ; s=1, \ldots, T)
$$



(4) $\sum_{s=1}^{j+t p_{q}} \sum_{t=s}^{\min \left(s+t d, j+t p_{q}\right)} v_{q s i t}+n v_{q j}=s t_{q, 1-j, 0}$
$\left(q=1, \ldots, Q ; j=1-t p_{q}, \ldots, 0 \mid s t_{q, 1-j, 0}>0\right)$
(5) $\sum_{s=\max (1, j-t d)}^{\min \left(j+t p_{q^{\prime}}, T\right)} \sum_{t=\max (j, s)}^{\min \left(j+t p_{q^{\prime}} s+t d, T\right)} v_{q s j t} \leq p_{q j}$
$(q=1, \ldots, Q ; j=1, \ldots, T)$
(6) $s t_{q 1 t}=p_{q t}-\sum_{s=\max (1, t-t d)}^{t} v_{q s t t}$
$(q=1, \ldots, Q ; t=1, \ldots, T)$
(7) $s t_{q j t}=s t_{q, j-1, t-1}-\sum_{s=\max (t-t d, 1)}^{t} v_{q, s, t-j+1, t}\left(q=1, \ldots, Q ; t=1, \ldots, T ; j=2, \ldots, t p_{q} \mid\left[s t_{q, j-t, 0}>0, \forall j \geq t+1\right]\right)$
(8) $\sum_{q=1}^{Q}\left(\frac{p_{q t}}{\rho_{q t}}+T P_{q} \cdot e_{q t}\right) \leq x_{t}$
(9) $p_{q t} \leq e_{q t} \cdot \rho_{q t} \cdot\left(h M_{t}-T P_{q}\right)$
$(q=1, \ldots, Q ; t=1, \ldots, T)$
(10) $\sum_{t=j-L+1}^{j} x_{t} \leq h_{L} \cdot L$
$\left(j=L, \ldots, T \mid\left(\exists i \mid[j-L+1, \ldots, j] \in S_{i}\right)\right)$
(11) $x_{t} \leq h_{A}+\left(h M_{t}-h_{A}\right) \cdot a_{t}$
(12) $\sum_{\forall t \in S_{i}} a_{t} \leq A_{i}$
$(\forall i \in W)$
(13) $x_{t} \leq h M_{t}+\left(h_{B}-h M_{t}\right) \cdot b_{t}$
(14) $\sum_{\forall t \in S_{i}} b_{t} \geq B_{i}$
$(\forall i \in W)$
(15) $y_{i} \leq M Y_{i}$
(16) $x_{t} \geq h m_{t}$
and
(17) $x_{t}, y_{i}, p_{q t}, s t_{q j t}, v_{q s j t}, r_{q t}, n v_{q s} \geq 0$
(18) $a_{t}, b_{t}, e_{q t} \in\{0,1\}$

The objective function to maximise, (1), is the profit (income minus costs due to overtime, temporary workers, product elimination, production, lost demand, and inventory). (2) expresses, for each worker, the annual balance of working hours. (3) expresses the served and required demand balance. (4) imposes that, if available inventory at the beginning of the planning horizon is not delivered before 
perishing, it is to be eliminated. (5) expresses, for each week, the balance of produced and delivered production. (6) imposes that the remaining production of a period is considered as inventory with an age of 1 week (when $t=T$, variables $s t_{q 1 T}$, corresponding to inventory at the end of the planning horizon are to be replaced with 0 ). (7) expresses the delivering and inventory balance (when $t=T$, variables $s t_{q j T}$ are to be replaced with 0). (8) imposes an upper bound on the production, depending on weekly working hours; it considers the average time (in hours) needed to prepare the process to produce product q. (9) imposes an upper bound on the production for each product (considering the preparation times of the process) and forces the binary variable $e_{q t}$ to take value 1 if product $q$ is produced in week $t$. (10) imposes the upper bound to the average weekly working hours for a subset of $L$ consecutive weeks (note that this constraint is included only if there exists at least one worker who has been working every week belonging to the set). If the team works a number of hours greater than $h_{A}$ in week $t$ (i.e., if it is a "strong" week), then (11) forces the binary variable, $a_{t}$, to take value 1. (12) imposes, for each worker, an upper bound for the number of weeks in which the worker is not on holiday and the binary variable, $a_{t}$, can take value 1 . Thus, (11) and (12) together impose the upper bound for "strong" weeks. If the team works a number of hours greater than $h_{B}$ in week $t$ (i.e., if it is not a "weak" week), then (13) forces the binary variable, $b_{t}$, to take value 0 . (14) imposes, for each worker, a lower bound for the number of weeks in which the worker is not on holiday and the binary variable $b_{t}$ can take value 1 . Thus, (13) and (14) together impose the lower bound for "weak" weeks. (15) imposes, for each worker, the upper bound for annual overtime. (16) imposes the lower bound for weekly working hours (note that the upper bound is included in (11) and (13)). And finally, (17) and (18) express the non-negative and binary character of the variables.

\section{Model AHNFH (annualised hours and non-fixed holidays)}

Considering holidays as a part of the model forces us to define a working-hour variable for each week and worker. Moreover, to take into account that the productivity for each product depends on the presence of temporary personnel, the hours dedicated to each product are to be considered (only when $Q>1$ ). Finally, binary variables $a_{t}$ and $b_{t}$ must be defined for each worker. Although the number of workers and products may be not very large, the size of the model, compared with the previous Model $A H F H$, is significantly increased

We make use of the additional following notation. 


\section{Data:}

Wmax maximum number of workers taking their holidays in any week.

$h w 1_{i}, h w 2_{i} \quad$ number of holiday weeks of the first and second holiday period respectively for worker $i(\forall i \in W)$

$t 1_{i}, t 2_{i} \quad$ first and last week respectively in which worker $i$ can take holidays corresponding to the first holiday period $(\forall i \in W)$

$t 3_{i}, t 4_{i} \quad$ first and last week respectively in which worker $i$ can take holidays corresponding to the second holiday period $(\forall i \in W)$

t1, $t 2$ first and last week respectively in which a worker can take holidays corresponding to the first holiday period; $t 1=\min \left(t 1_{i}\right)$ and $t 2=\max \left(t 2_{i}\right)$.

t3, $t 4$ first and last week respectively in which a worker can take holidays corresponding to the second holiday period; $t 3=\min \left(t 3_{i}\right)$ and $t 4=\max \left(t 4_{i}\right)$.

$\sigma_{q t} \quad$ productivity (in units/employee_hour) for product $q$ in week $t(\mathrm{q}=1, \ldots, Q ; \mathrm{t}=1, \ldots, T)$.

$\mu_{q t} \quad$ productivity (in units/temporary_hour) for product $q$ in week $t(\mathrm{q}=1, \ldots, \mathrm{Q} ; \mathrm{t}=1, \ldots, T)$.

\section{Variables:}

$u_{q t} \quad$ non-negative real variable that indicates the number of working hours in week $t$ dedicated to product $q$, including setup times $(\mathrm{q}=1, \ldots, Q ; \mathrm{t}=1, \ldots, T)$. Therefore, the number of working hours in a week is the sum of these variables for each product.

$w_{\text {qit }} \quad$ non-negative real variable that indicates the number of working hours in week $t$ for worker $i,(\mathrm{q}=1, \ldots, Q ; \forall i \in W ; \mathrm{t}=1, \ldots, T)$.

$a_{i t} \in\{0,1\} \quad$ if the number of working hours for worker $i$ in week $t$ is greater than $h_{A}$ (the week is "strong”), then these variables take value $1(\forall i \in W ; \mathrm{t}=1, \ldots, T)$. This variable has the same meaning as the corresponding $a_{t}$.

$b_{i t} \in\{0,1\} \quad$ if the number of working hours for worker $i$ in week $t$ is greater than $h_{B}$ (the week is not "weak"), then these variables take value $0(\forall i \in W ; \mathrm{t}=1, \ldots, T)$.

$v c 1_{i t} \in\{0,1\} \quad$ Indicates whether employee $i$ starts his first holiday period in week $t(\forall i \in W$, $\left.\mathrm{t}=t 1_{i}, \ldots, t 2_{i}-h w 1_{i}+1\right)$. 
$v c 2_{i t} \in\{0,1\} \quad$ Indicates whether employee $i$ starts his second holiday period in week $t(\forall i \in W$, $\left.\mathrm{t}=t 3_{i}, \ldots, t 4_{i}-h w 2_{i}+1\right)$.

\section{Model :}

It is possible to build $A H N F H$ from $A H F H$ by adding some constraints and modifying some others. The changes are described below:

- In Equation (1), $C T \cdot\left(|W| \cdot \sum_{q=1}^{Q} u_{q t}-\sum_{\forall i \in W} \sum_{q=1}^{Q} w_{q i t}\right)$ is included instead of $\left(C T \cdot \sum_{t=1}^{T} N T_{t} \cdot x_{t}\right)$ to model the temporary worker cost.

- In Equation (2), $\left(\sum_{t=1}^{T} \sum_{q=1}^{Q} w_{\text {qit }}\right)$ is included instead of $\left(\sum_{\forall t \in S_{i}} x_{t}\right)$ to compute the amount of total working hours of worker $i(\forall i \in W)$.

- Equations (19) and (20) are included to ensure that each worker takes his holidays:

(19) $\sum_{t=t 1_{i}}^{t 2_{i}-h w 1_{i}+1} v c 1_{i t}=1$

(20) $\sum_{t=t 3_{i}}^{t 4_{i}-h w 2_{i}+1} v c 2_{i t}=1$

- Equations (21) to (26) are included to ensure that all present employees carry out the same working weeks (and of course all workers dedicate the same number of hours to a product). The sum of $v c 1_{i j}$ and $v c 2_{i j}$ variables appearing in Equations (22), (23), (25) and (26) show whether the worker is on holiday (if it takes value 1 ) or not (if it takes value 0 ):

(21) $w_{q i t}=u_{q t} \quad\left(q=1, \ldots, Q ; \forall i \in W ; t=1, \ldots, T \mid t \notin\left(\left[t 1_{i}, \ldots, t 2_{i}\right] \vee\left[t 3_{i}, \ldots, t 4_{i}\right]\right)\right)$

(22) $w_{q i t} \leq h M_{t} \cdot\left(1-\sum_{j=\max \left(t 1_{1}, t-h w 1_{i}+1\right)}^{\min \left(t, t 2_{i}-h w 1_{i}+1\right)} v c 1_{i j}\right) \quad\left(q=1, \ldots, Q ; \forall i \in W ; t=t 1_{i}, \ldots, t 2_{i}\right)$

(23) $u_{q t} \leq w_{q i t}+h M_{t} \cdot\left(\sum_{j=\max \left(t 1_{i}, t-h w 1_{i}+1\right)}^{\min \left(t, t 2_{i}-h w 1_{i}+1\right)} v c 1_{i j}\right) \quad\left(q=1, \ldots, Q ; \forall i \in W ; t=t 1_{i}, \ldots, t 2_{i}\right)$

(24) $w_{q i t} \leq u_{q t} \quad\left(q=1, \ldots, Q ; \forall i \in W ; t=t 1_{i}, \ldots, t 2_{i} ; t=t 3_{i}, \ldots, t 4_{i}\right)$ 
(25) $w_{q i t} \leq h M_{t} \cdot\left(1-\sum_{j=\max \left(t 3_{i}, t-h w 2_{i}+1\right)}^{\min \left(t, t 4_{i}-h w 2_{2}+1\right)} v c 2_{i j}\right)$

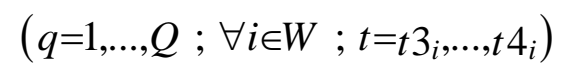

(26) $u_{q t} \leq w_{q i t}+h M_{t} \cdot\left(\sum_{j=\max \left(t 3_{i}, t-h w 2_{i}+1\right)}^{\min \left(t, t 4_{i}-h w 2_{i}+1\right)} v c 2_{i j}\right)$

- Equations (27) and (28) are included to impose the lower bound for the number of nontemporary workers (which is the same as imposing an upper bound for the number of temporary workers):

(27) $\sum_{\forall i \in W} \sum_{\mid t \in\left[11_{i}, t 2_{i}\right]} \sum_{j=\max \left(t 1_{i}, t-h w 1_{i}+1\right)}^{\min \left(t, t 2_{i}-h w 1_{i}+1\right)} v c 1_{i j} \leq W m a x$
(28) $\sum_{\forall i \in W} \sum_{\mid t \in\left[t 3_{i}, t 4_{i}\right]} \sum_{j=\max \left(t+t 3_{i}, t-h w 2_{i}+1\right)}^{\left.t 4_{i}-h w 2_{i}+1\right)} v c 2_{i j} \leq W m a x$

- Equations (8) and (9) are replaced with (29), (30) and (31):

(29) $p_{q t} \leq \sigma_{q t} \cdot|W| \cdot\left(u_{q t}-T P_{q} \cdot e_{q t}\right) \quad(q=1, \ldots, Q ; t=1, \ldots, T \mid t \notin([t 1, \ldots, t 2] \vee[t 3, \ldots, t 4]))$

(30) $p_{q t} \leq \sigma_{q t} \cdot \sum_{\forall i \in W}\left(w_{q i t}-T P_{q} \cdot e_{q t}\right)+\mu_{q t} \cdot\left(|W| \cdot\left(u_{q t}-T P_{q} \cdot e_{q t}\right)-\sum_{\forall i \in W}\left(w_{q i t}-T P_{q} \cdot e_{q t}\right)\right)$

$$
(q=1, \ldots, Q ; t=t 1, \ldots, t 2 ; t=t 3, \ldots, t 4)
$$

(31) $p_{q t} \leq e_{q t} \cdot \sigma_{q t} \cdot|W| \cdot\left(h M_{t}-T P_{q}\right)$

$$
(q=1, \ldots, Q ; t=1, \ldots, T)
$$

- Equation (10) is replaced with (32):

(32) $\sum_{t=j-L+1}^{j} \sum_{q=1}^{Q} u_{q t} \leq h_{L} \cdot L$

- Equations (11) and (12) are replaced with (33) and (34):

(33) $\sum_{q=1}^{Q} w_{\text {qit }} \leq h_{\mathrm{A}}+\left(h M_{t}-h_{\mathrm{A}}\right) \cdot a_{i t}$

(34) $\sum_{t=1}^{T} a_{i t} \leq A_{i}$

- Equations (13) and (14) are replaced with (35) and (36): 

(35) $\sum_{q=1}^{Q} w_{q i t} \leq h M_{t}+\left(h_{B}-h M_{t}\right) \cdot b_{i t}$
$(\forall i \in W ; t=1, \ldots, T)$
(36) $\sum_{t=1}^{T} b_{i t} \geq B_{i}$

- Equation (16) is replaced with (37):

(37) $\sum_{q=1}^{Q} u_{q t} \geq h m_{t}$

- Finally, Equations (17) and (18) are replaced with (38) and (39):

(38) $u_{q t}, w_{q i t}, y_{i}, p_{q t}, s t_{q j t}, v_{q s j t}, r_{q t}, n v_{q s} \geq 0$

(39) $a_{i t}, b_{i t}, e_{q t}, v c 1_{i t}, v c 2_{i t} \in\{0,1\}$

- The rest of the model (Equations (3), (4), (5), (6), (7) and (15)) remains invariable.

\section{Production planning under annualised hours (all workers take their holidays at the same time)}

In many companies all workers take their holidays at the same time and production is then stopped (this not precluding, obviously, that may be demand at all periods, even at those corresponding to holidays). According to these assumptions, there is no need of hiring temporary personnel to stand in for employees.

Again, holiday weeks may be considered as previously agreed or to be determined, giving rise to the AHFSH model (annualised hours and fixed simultaneous holidays) and the AHNFSH model (annualised hours and non-fixed simultaneous holidays) respectively. Formulating these models is straightforward once the AHFH and AHNFH models have been understood.

First of all, assuming simultaneous holidays means that it is not necessary to consider each worker separately: they can be considered as a team. Thus, there are no variables to be individualised (for example, $y$ must be defined instead of $y_{i}$ ). Secondly, the company does not hire temporary personnel and the production is stopped when the team takes its holidays. It is therefore unnecessary to define 
the additional variables $u_{q t}$ and $w_{i q t}$ when holidays are not fixed beforehand (it is sufficient to define $x_{t}$ variables) and the size of the model is not excessively increased.

To model AHFSH one can start from AHFH, but taking into account that all workers take their holidays in the same weeks and that during these weeks there is no production but there is demand. Variables and constraints about temporary workers are deleted.

Once the AHFSH model has been formulated, only the variables and constraints concerning holidays, which are included below, must be added to obtain the AHNFSH model. Note that holiday variables are the same as those defined for the $A H N F H$ model, but without considering workers individually.

$$
\begin{aligned}
& \text { (40) } \sum_{t=t 1}^{t 2-h w 1+1} v c 1_{t}=1 \\
& \text { (41) } \sum_{t=t 3}^{t 4-h w 2+1} v c 2_{t}=1 \\
& \text { (42) } x_{t} \leq h M_{t} \cdot\left(1-\sum_{j=\max (t 1, t-h w 1+1)}^{\min (t, t 2-h w 1+1)} v c 1_{j}\right) \\
& \text { (43) } x_{t} \geq h m_{t} \cdot\left(1-\sum_{j=\max (t 1, t-h w 1+1)}^{\min (t, t 2-h w 1+1)} v c 1_{j}\right) \\
& \text { (44) } x_{t} \leq h M_{t} \cdot\left(1-\sum_{j=\min (t, t 4-h w 2+1)}^{\sum_{\min (t, t, t-t-h w 2+1)}} v c 2_{j}\right) \\
& \text { (45) } x_{t} \geq h m_{t} \cdot\left(1-\sum_{j=\max (t 3, t-h w 2+1)} v c 2_{j}\right)
\end{aligned}
$$

\section{Computational experiment}

A computational experiment was performed to evaluate the efficiency of the MILP models and the main results of annualising working time (comparing the results obtained for different situations). Overall, we consider that these results are very satisfactory.

The basic data used for the experiment are as follows: 
- $\quad T=52$ (considering 48 working weeks and 4 holiday weeks).

- $|W|=25$ workers.

- $Q=1$ and $Q=5$ products.

- $T P_{q}=0$ (thus, variables $e_{q t}$ are not in the model).

- In all instances, the annual number of holiday weeks is constrained to be equal to four, distributed into two non-interrupted parts of one and three weeks respectively. For the AHFH and AHFSH models, the temporary location of holidays was fixed at random (the week of the first period, in winter, and the three weeks of the second period, in summer). This way of generating holiday weeks might be not very realistic, but it does not make solving the models any easier.

- To evaluate different situations under which one would establish a negotiation framework, three levels of $H_{i}$ were considered, depending on the reduction in working time as a compensation for the worsening of working conditions: 1,920 hours (corresponding to an average of 40 hours/week); 1,824 hours (corresponding to an average of 38 hours/week); and 1,680 hours (corresponding to an average of 35 hours/week).

- Also, four different lower and upper bounds were considered for weekly working hours, depending on the weekly flexibility accepted by the workers: $[40,40]$ (which corresponds to a non-AH situation without overtime and is only considered when $H=1,920$ hours/year); [40, 50] (which might correspond to a non-AH situation with weekly overtime and is only considered when $H=1,920$ hours/year); [30, 45]; and [25, 50].

- $\quad L=12$ weeks; $h_{L}=44$ hours/week (when possible; not for [40, 40])

- $A_{i}=15$ weeks; $h_{A}=44$ hours/week (when possible; not for $[40,40]$ )

- $\quad B_{i}=10$ weeks; $h_{B}=30$ hours/week (when possible; not for $[40,40]$ or $[40,50]$ )

- There are three different demand patterns throughout the year: Demand Type 1 corresponds to a non-seasonal capacity pattern (the required capacity is the same for every week in the planning horizon); Demand Type 2 corresponds to a seasonal pattern with one peak; and Demand Type 3 corresponds to a seasonal pattern with two peaks. In each case, total demand is approximately equal to total capacity. Figures 1, 2 and 3 show the shapes of these patterns. Furthermore, the peaks can be more or less pronounced; three levels were considered (small peaks, medium peaks and high peaks).

Figure 1. Type of demand 1 (no seasonality) 
Figure 2. Type of demand 2 (one peak)

\section{Figure 3. Type of demand 3 (two peaks)}

The efficiency of the models was tested using the ILOG CPLEX 8.1 optimiser (a maximum solving time of 1,800 seconds and a relative and absolute gap of 0.01 were set) and a PC Pentium IV at 1.80 GHz with $512 \mathrm{Mb}$ RAM. A total amount of 864 instances for the AHFH and AHNFH models and 192 instances for the AHFSH and AHNFSH models, which were obtained as follows, were solved:

- $\quad$ For $Q=1$ (models $A H F H$ and $A H N F H)$ :

o 3 types of demand.

o 3 levels of peak demands (only for the second and third types of demand).

o 3 levels of inventory holding costs (low, medium and high).

o 2 levels for the maximum delay to serve the demand (low and high).

o 2 levels for the maximum time products can be stored.

o 8 combinations for annual hours and the lower and upper bounds for weekly working hours.

- $\quad$ For $Q=5$ (models AHFH, AHNFH, AHFSH and AHNFSH):

o 2 types of demand.

o 3 levels of peak demand.

o 2 levels for the maximum delay to serve the demand (low and high).

o 2 levels for the maximum time products can be stored.

o 8 combinations for annual hours and the lower and upper bounds for weekly working hours.

Table 1 shows the main results regarding solving times: \% of instances solved to optimality before achieving the maximum solving time set to 1,800 seconds (\%Opt) and, for those instances, minimum solving time $\left(t_{\min }\right)$, average solving time $(\bar{t})$ and maximum solving time $\left(t_{\max }\right)$. The size of the instances is also included: number of binary variables ( $\mathrm{n}^{\circ}$ bin var), average number of real variables ( $\mathrm{n}^{\mathrm{o}}$ real var) and average number of constraints ( $\mathrm{n}^{\mathrm{o}}$ const). Table 2 shows a comparison between the 
objective function obtained by the two models. It includes the \% of instances in which the solution of the $A H N F H$ model is equal or very similar to ( $\% \mathrm{z}$ similar), better ( $\% \mathrm{z}$ better) or worse ( $\% \mathrm{z}$ worse) than the one obtained by solving the $A H F H$ model to optimality. It also includes the minimum (\% $\left.I_{\min }\right)$, average $(\% \bar{I})$ and maximum (\% $\left.I_{\max }\right)$ improvement in the objective function obtained by allowing the holidays to be fixed by the model (AHNFH compared to AHFH).

The models for fixed and/or simultaneous holidays (AHFH, AHFSH and AHNFSH) are always solved to optimality in a very short time (few seconds), while introducing holidays as variables when workers do not take their holidays at the same time creates a great difficulty in solving the model (AHNFH). With the parameters used and for $Q=5$, failing to obtain the optimal solution means that in some cases the solution of the AHNFH model is considerably worse than the optimal solution of the AHFH model. Nevertheless, it is possible (and likely) that profit will be increased by solving the AHNFH model, even if there is insufficient time to solve it to optimality.

Table 1. Computing times (in seconds) for optimality solved instances (maximum solving time set to 1,800 seconds)

Table 2. Comparison of objective function: $A H N F H$ vs $A H F H$ and $A H N F S H$ vs $A H F S H$

To show the benefits of annualising working hours, two optimal solutions of the AHFH model are compared. Figure 4 shows production, inventory levels, lost demand and how demand is covered (without or with delay) in a situation without flexibility and without overtime. Figure 5 shows the same information when annualised hours are introduced. The increase in profit is mainly due to the reduction in lost and deferred demand. In Figure 5 one can see how lost demand can be reduced (and even eliminated) and how the demand is almost always served on time due to the flexibility provided by $\mathrm{AH}$, even when annual working time is reduced.

In addition to the increase in profit (which depends on the particular parameters), it is possible to compare the two situations from a qualitative point of view. The company represented in Figure 4 defers the demand in a large number of weeks, ruining its image and therefore diminishing its competitiveness. On the other hand, thanks to $\mathrm{AH}$, the majority of customers of the company represented in Figure 5 are served on time and this situation is therefore more favourable to competitiveness. Finally, the use of temporary workers (in hours) decreases because it is possible to 
plan short hours when workers are on holidays. If the intervention of temporary personnel in the process leads to a product of less quality, then quality is increased through flexibility.

As an example, by analysing the results obtained solving the $A H F H$ model to optimality (for $Q=1$ product), one can see that with the specific parameters used in the computational experiment an increase in flexibility - without a reduction in working time - produces an average decrease of $25 \%$ in the temporary personnel cost and a $100 \%$ reduction in lost demand.

Figure 4. AHFH Model. Production, inventory, lost demand and covered demand (H= 1920 hours/year; $[h m, h M]=[40,40]$ hours/week; $t d=2$ weeks; $\left.Q=1 ; z^{*}=K\right)$

Figure 5. AHFH Model. Production, inventory, lost demand and covered demand (H= 1824 hours/year; $[h m, h M]=[25,50]$ hours/week; $t d=2$ weeks; $\left.Q=1 ; z^{*}=1.16 \cdot \mathrm{K}\right)$

In most countries companies cannot introduce irregular working hours if workers do not agree, so the question is whether workers will really accept an increase in flexibility. Besides the convincing argument of conserving their jobs even in periods of low demand, companies should offer some kind of compensation that will lead workers to accept more or less flexibility. Hence, it is essential to have a tool to help in the bargaining.

When holidays are fixed beforehand or workers take them simultaneously, optimally solving the model requires an insignificant time. Thus, the company could solve several scenarios in a short time and then establish the framework for collective bargaining.

Solving the model for different levels of flexibility and working time reduction provides the company and the workers with the information that they require before agreeing to the conditions of the annualised hours system. Table 3 shows the results of a specific case, where $Q=1$ product and workers do not take their holidays simultaneously ( $K$ is the profit obtained in a situation without flexibility and without a reduction in working time). The company can see how the profit increases when flexibility is high, even when reducing working time.

Table 3. Example of decisions (weekly flexibility and annual hours) that result in an increase in the profit. 
Two methods for increasing the profit by implementing annualised hours might be as follows: (1) by increasing weekly flexibility and reducing working time as a compensation for the workers; or (2), by increasing flexibility and not reducing working time but instead offering financial compensation to the workers. The company and the workers can agree on satisfactory conditions for both.

Once AH system conditions have been agreed, the company can try to convince the workers (or some of them) to determine their holidays by using the second model and, depending on the increase in the profit, offer them an economic compensation.

\section{Conclusions}

Annualising working hours is a competitive way of adjusting productive capacity to seasonal demand and of obtaining flexibility in the distribution of annual working hours. Thanks to AH the company is able to reduce the use of temporary workers and thus improve quality and productivity. Also, adapting capacity to demand enables the company to serve their customers on time, improving the company's image and making it more competitive.

Annualising entails new problems to be faced, particularly those associated with planning both production and the weekly number of working hours on an annual scale while respecting the constraints established by law and those laid down in collective bargaining agreements in order to take into account working conditions. This paper presents a specific problem of planning working hours (including holidays) and production in industries in which products are perishable. It also presents two optimisation models for solving the problem. A computational experiment showed that MILP is an efficient tool for solving the problem in an optimal way, especially in those situations (the most typical) in which holidays are fixed beforehand or workers take their holidays at the same time.

One of the main drawbacks of $\mathrm{AH}$ is that it may result in a worsening in the workers' working conditions, so they usually do not accept such schemes in exchange for nothing. Considering different levels for annual working hours (with or without a reduction in working time) and different levels for weekly flexibility when solving the problem allows the company to provide itself and the workers with adequate information for negotiating the conditions of an annualised hours system. The company 
will then see how profit increases when flexibility is high, even when working time is reduced or financial compensation is offered to the workers for the worsening of their working conditions.

It can be concluded that the proposed models are a very useful management tool to plan working time and to help in the bargaining process. 


\section{Notes}

* Supported by the Spanish MCyT projects DPI2001-2176 and DPI2004-05797, co-financed by FEDER. 


\section{References}

C. Azmat; M. Widmer. (2004a). A case study of single shift planning and scheduling under annualized hours: A simple three step approach. European Journal of Operational Research, 153, 1, 148-175

C. Azmat; T. Hürlimann; M. Widmer. (2004b). Mixed Integer Programming to Schedule a Single-Shift Workforce under Annualized Hours. Annals of Operation Research, 128, 199-215

P. Chan; G. Weil. (2002). Using Multiple-level Models to solve large-scale employee scheduling. Paper presented at European Conference for Artificial Intelligence 2002; July 21-26 2002, Lyon, France.

D. Clutterbuck. (1982). After flexible hours, now it’s flexiyears. International Management, 37 (3), 31-36.

A. Corominas; A. Lusa; R. Pastor. (2002). Using MILP to plan annualised hours. Journal of the Operational Research Society, 53 (10), 1101-1108.

A. Corominas; A. Lusa; R. Pastor. (2004a). Characteristics and classification of the annualised working hours planning problems. International Journal of Services Technology and Management, 5 (5/6), 435-447.

A. Corominas; A. Lusa; R. Pastor. (2004b). Planning annualised hours with a finite set of weekly working hours and joint holidays. Annals of Operations Research, 128, 217-233.

A. Corominas; A. Lusa; R. Pastor. (2005a). Annualised hours: A real flexibility tool. ORInsight, 18 (1), 10-14.

A. Corominas; A. Lusa; R. Pastor. (2005b). Using a MILP model to establish a framework for an annualised hours agreement. European Journal of Operational Research (in press, available on-line).

P. Curran. (1992). Annual hours brings productivity boost to Spicers. Management Services, July, 3233.

E.V. Gonçalves; J.A., Marçola. (2001). Annualized hours as a capacity planning tool in make-to-order or assemble-to-order environment: an agricultural implements company case. Production Planning \& Control, 12, (4), 388-398.

R. Hung. (1999a). A multiple-shift workforce scheduling model under annualized hours. Naval Research Logistic, 46 (6), 726-736.

R. Hung. (1999b). Scheduling a workforce under annualized hours. International Journal of Production Research, 37 (11), 2419-2427.

P. Lynch. (1985). Annual Hours: An idea whose time has come. Personnel Management, November, 46-50. 
J. MacMeeking. (1995). Why Tesco's new composite distribution needed annual hours. International Journal Retail Distribution Management, 23 (9), 36-38.

L. Mazur. (1995). Coming: the annual workweeks. Across the Board, 32 (4), 42-45.

MES: Ministère de l'Emploi et de la Solidarité (2004): www.35h.travail.gouv.fr. 03/28/2004. France. 
Table 1. Computing times (in seconds) for optimality solved instances (maximum solving time set to 1,800 seconds)

\begin{tabular}{|c|c|c|c|c|c|c|c|c|}
\hline Model & $Q$ & $t_{\min }$ & $\bar{t}$ & $t_{\max }$ & $\% O p t$ & $\mathrm{n}^{\mathrm{o}}$ bin var & $\mathrm{n}^{0}$ real var & $\mathrm{n}^{\mathrm{o}}$ const \\
\hline \multirow{2}{*}{ AHFH } & 1 & \multirow{2}{*}{\multicolumn{3}{|c|}{ insignificant }} & 100 & 104 & 3,129 & 976 \\
\hline & 5 & & & & 100 & 104 & 15,337 & 3,512 \\
\hline \multirow{2}{*}{ AHNFH } & 1 & 2.31 & 19.67 & 1,045 & 14.2 & 3,150 & 4,429 & 6,053 \\
\hline & 5 & - & - & - & 0 & 3,150 & 22,045 & 18,797 \\
\hline AHFSH & 5 & \multicolumn{3}{|c|}{ insignificant } & 100 & 96 & 11,460 & 3,468 \\
\hline AHNFSH & 5 & \multicolumn{3}{|c|}{ insignificant } & 100 & 126 & 13,269 & 3,518 \\
\hline
\end{tabular}


Table 2. Comparison of objective function: $A H N F H$ vs $A H F H$ and $A H N F S H$ vs $A H F S H$

\begin{tabular}{|c|c|c|c|c|c|c|c|}
\hline Model & $Q$ & $\% \mathrm{z}$ similar & $\% \mathrm{z}$ better & $\% \mathrm{z}$ worse & $\% I_{\min }$ & $\% \bar{I}$ & $\% I_{\max }$ \\
\hline AHNFH vs AHFH & 1 & 24 & 76 & 0 & 0 & 8.8 & 164.5 \\
\hline AHNFH vs AHFH & 5 & 23 & 58 & 18 & -95.5 & 10 & 140 \\
\hline AHNFSH vs AHFSH & 5 & 52.3 & 47.7 & 0 & 0 & 2.64 & 85.5 \\
\hline
\end{tabular}


Table 3. Example of decisions (weekly flexibility and annual hours) that result in an increase in the profit. AHFH Model.

\begin{tabular}{|c|c|c|c|c|c|}
\cline { 3 - 6 } \multicolumn{1}{c|}{} & \multicolumn{5}{c|}{$[h m, h M]$ (hours/week) } \\
\cline { 2 - 7 } \multicolumn{1}{c|}{} & $\mathrm{z}^{*}$ (profit) & {$[40,40]$} & {$[40,50]$} & {$[30,45]$} & {$[25,50]$} \\
\hline \multirow{3}{*}{$H$} & 1,920 & $\mathrm{~K}$ & $1.016 \cdot \mathrm{K}$ & $1.46 \cdot \mathrm{K}$ & $1.55 \cdot \mathrm{K}$ \\
\cline { 2 - 6 } (hours/year) & 1,824 & & & $1.45 \cdot \mathrm{K}$ & $1.53 \cdot \mathrm{K}$ \\
\cline { 2 - 6 } & 1,680 & & & $1.021 \cdot \mathrm{K}$ & $1.083 \cdot \mathrm{K}$ \\
\hline
\end{tabular}




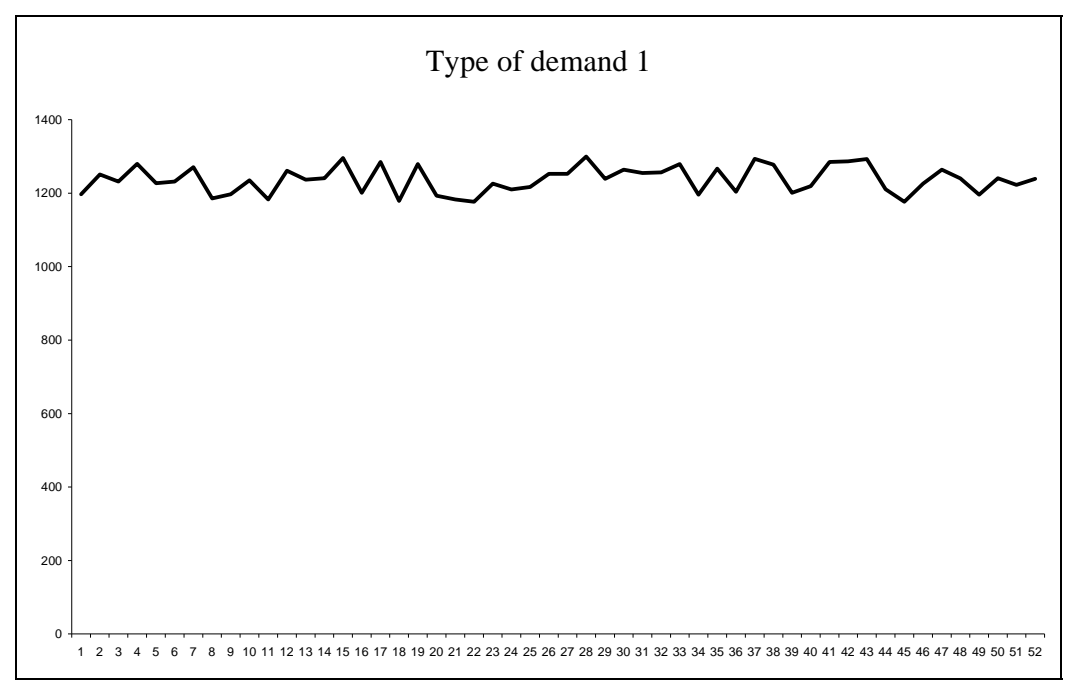

Figure 1. Type of demand 1 (no seasonality) 


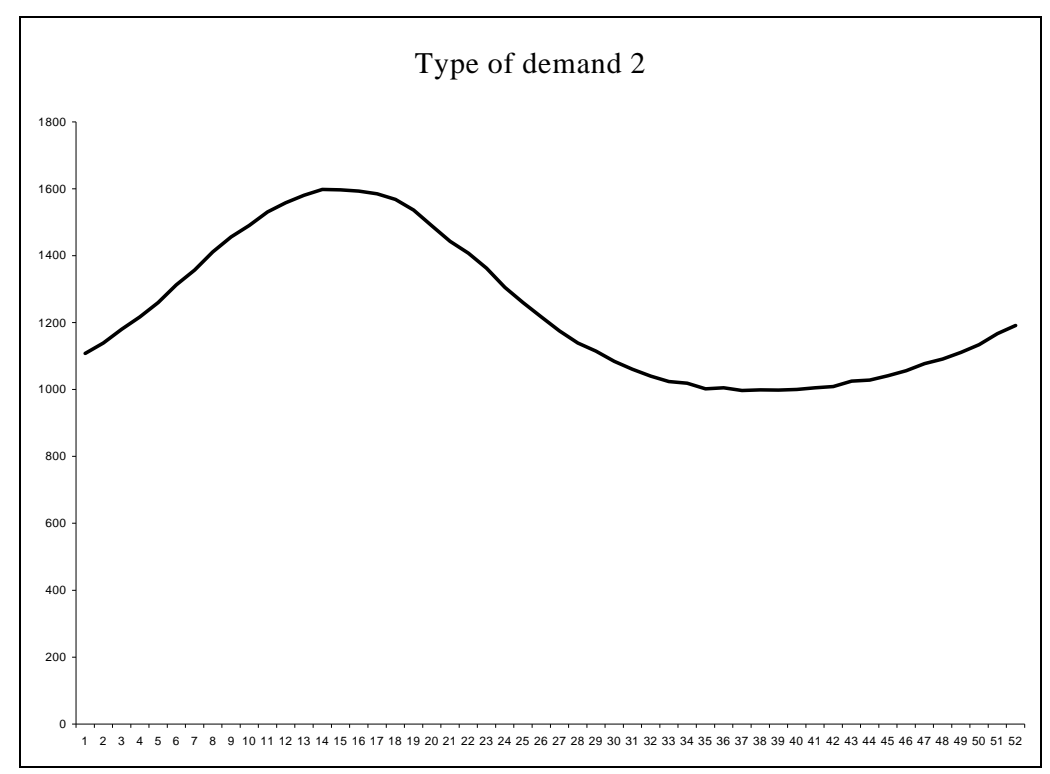

Figure 2. Type of demand 2 (one peak) 


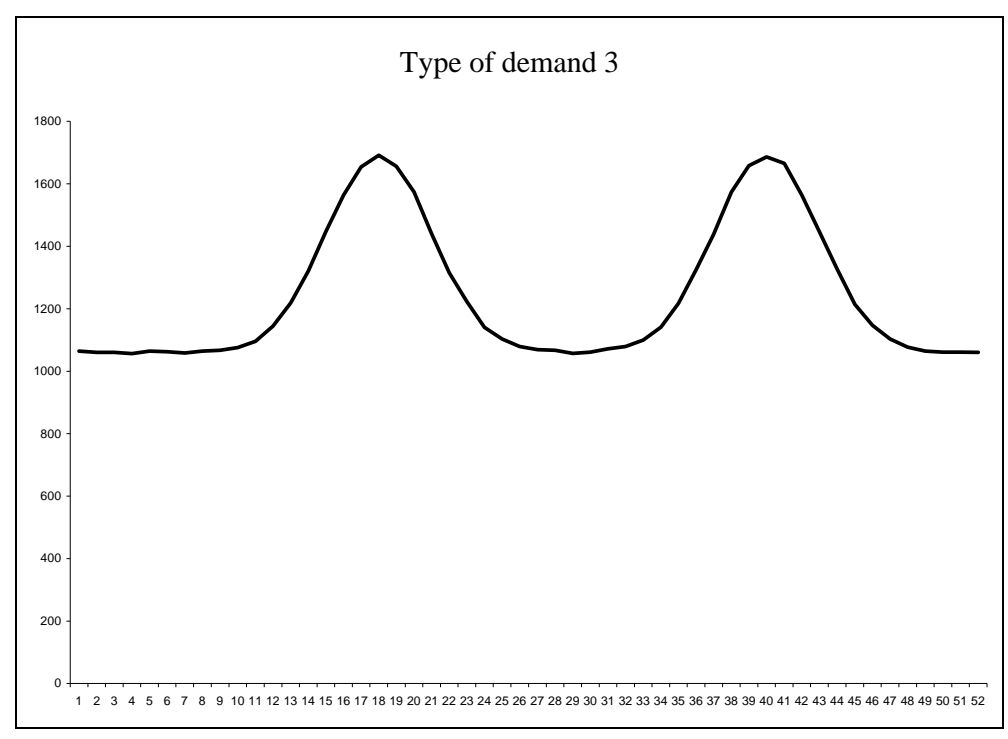

Figure 3. Type of demand 3 (two peaks) 


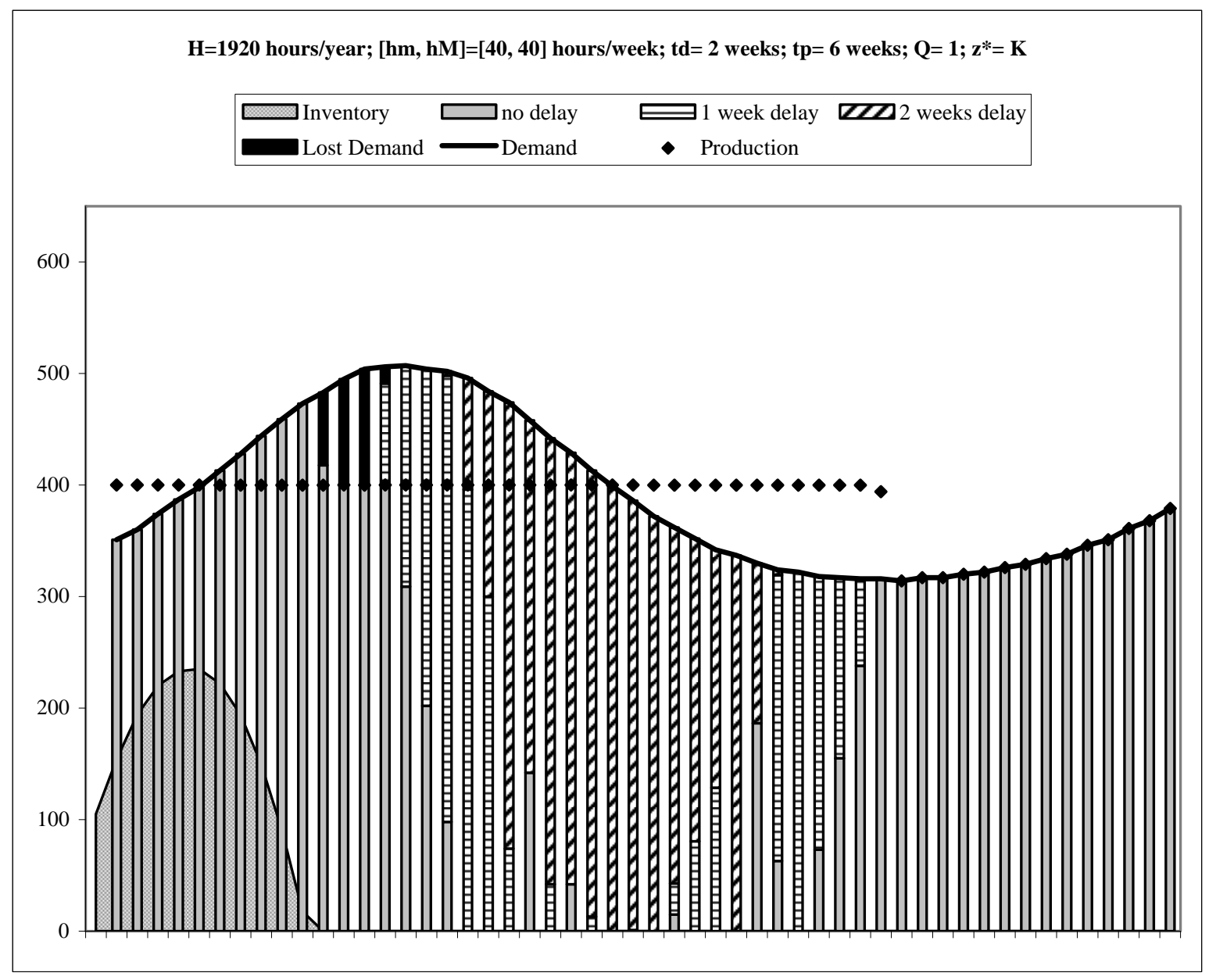

Figure 4. AHFH Model. Production, inventory, lost demand and covered demand $(H=1920$ hours/year; $[h m, h M]=[40,40]$ hours/week; $t d=2$ weeks; $Q=1 ; \mathrm{z}^{*}=\mathrm{K}$ ) 


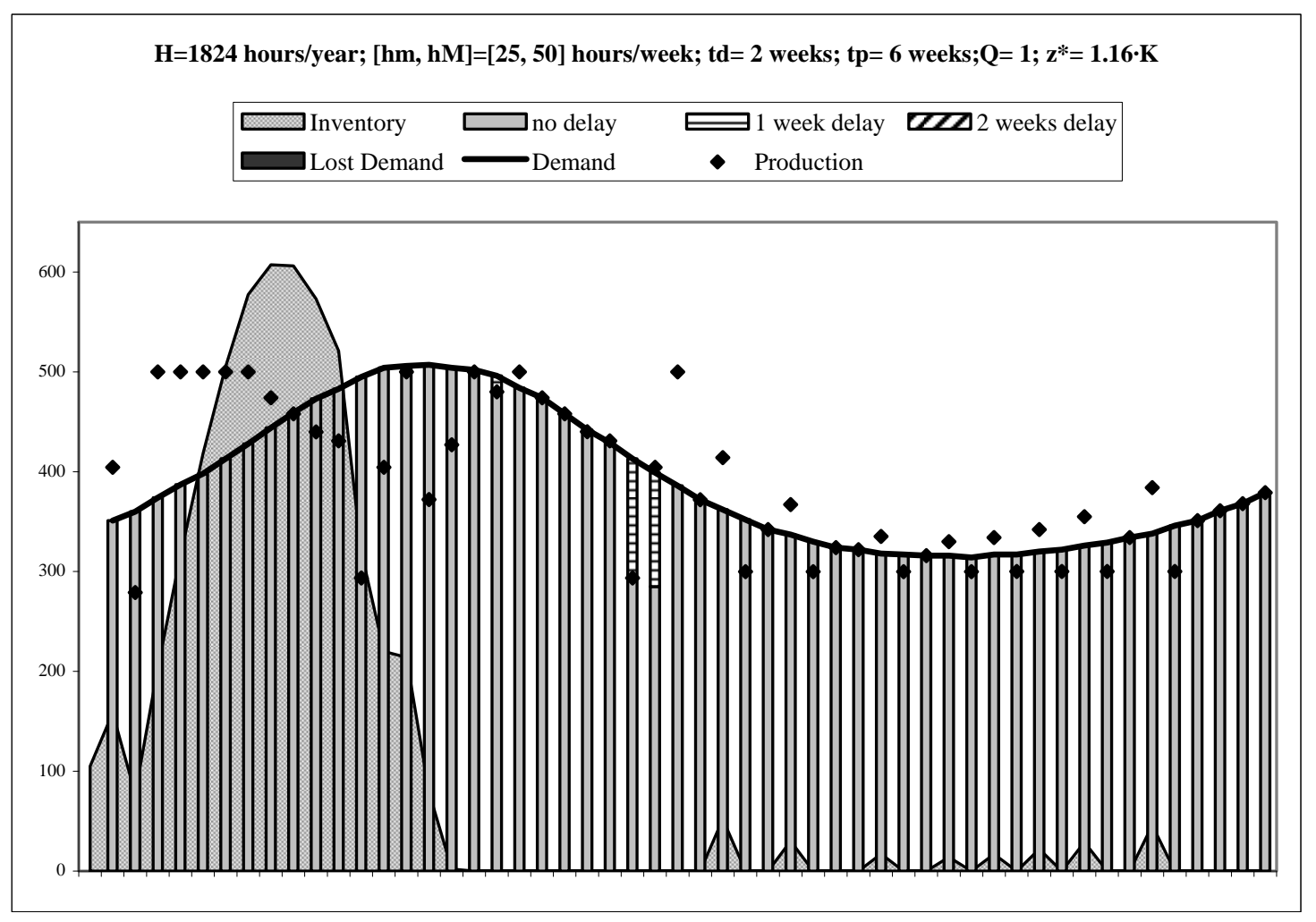

Figure 5. AHFH Model. Production, inventory, lost demand and covered demand ( $H=1824$ hours/year; $[h m, h M]=[25,50]$ hours/week; $t d=2$ weeks; $\left.Q=1 ; z^{*}=1.16 \cdot \mathrm{K}\right)$ 